\title{
ERASE: a novel nucleic-acid based antiviral mechanism
}

\author{
George Fu Gao (D ${ }^{1,2 凶}$ \\ (c) CEMCS, CAS 2021 \\ Cell Research (2021) 31:1142-1143; https://doi.org/10.1038/s41422-021-00568-9
}

\begin{abstract}
Nucleic acid-based antiviral systems, including CRISPR/Cas and RNA interference, play important roles in antiviral defense. In a paper recently published in Cell Research, Wu et al. revealed a novel nucleic acid-based antiviral mechanism against RNA viruses in mouse embryonic stem cells, which relies on the endogenous reverse transcriptase to produce viral complementary DNA and then RNase $\mathrm{H} 1$ to destroy the viral RNA.
\end{abstract}

In nature, life has evolved different types of antiviral immune mechanisms to combat viral infection, which can be divided into two major categories: the protein-based antiviral mechanism and the nucleic acid-based antiviral mechanism. ${ }^{1}$ Antibody and $\mathrm{T}$ cell-based immunity as well as interferon pathway are the representatives of the protein-based antiviral mechanism, ${ }^{2}$ while CRISPR/Cas and RNA interference (RNAi) are the representatives of the nucleic acid-based antiviral mechanisms. Over the past twenty years, nucleic acid-based antiviral systems have come into the spotlight. CRISPR/Cas in bacteria and archaea can integrate the DNA fragments of DNA bacteriophages into their genomes, which are named as clustered regularly interspaced short palindromic repeats (CRISPR). ${ }^{3}$ These phage-derived DNA fragments are transcribed into trans-activating CRISPR RNAs (tracrRNAs), which guide the Cas proteins to destroy the DNA of the homologous bacteriophages during subsequent infections. For RNAi in plants and insects, the double-stranded RNA intermediates of virus replication are processed into short interfering RNAs (siRNAs) by the RNase III enzyme Dicer and then the siRNAs are loaded into the effector complex RNAinduced silencing complex (RISC) to guide Argonaute protein 2 (Ago2) to cleave viral RNA genome. ${ }^{4}$

In the study reported by $\mathrm{Wu}$ and colleagues, a new type of nucleic acid-based antiviral pathway in mouse embryonic stem cells (mESCs) was discovered. ${ }^{5}$ Ancient retrovirus infection and integration leads to formation of thousands of retroviral sequence loci known as endogenous retroviruses (ERVs) in mammalian cells. ${ }^{6}$ Some of these ERVs keep intact open reading frames and encode active viral proteins including reverse transcriptase (RTase), but their functions are largely unknown. The authors first measured the activity of endogenous RTase in different cell types. They found that the RTase is more active in mESCs than somatic cells and its activity decreased dramatically along with ESC differentiation. They further showed that the viral DNA (vDNA) was generated by the RTase from non-retroviral RNA viruses (e.g., encephalomyocarditis picornavirus and mouse hepatitis coronavirus) in mESCs. Inhibiting the endogenous RTase activity by azidothymidine significantly promoted virus replication, while increasing the RTase activity by GSK-LSD1 suppressed virus replication in mESCs. Moreover, the authors showed that the antiviral function of RTase in mESCs is independent of RNAi and interferon pathway.

The authors further investigated the nature of the vDNA. Cell fractionation revealed that the vDNA is mainly localized in the cytoplasm. The DNase digestion experiments showed that the vDNA is sensitive to $\mathrm{S} 1$ nuclease but resistant to dsDNA restriction endonucleases, implying that the vDNA is single stranded. The unidirectional primer extension following restrictive endonuclease digestion further demonstrated that the vDNA is mainly composed of negative-sense ssDNA which is complementary to the viral genomic RNA. Immunofluorescent staining and DRIP experiments with monoclonal antibody S9.6 showed that the vDNA forms DNA/RNA hybrid with viral RNA in the infected cells. The authors further showed that the DNA/RNA hybrid was co-localized with RNase $\mathrm{H} 1$ in the cytoplasm and the vDNA could be pulled down by anti-RNase $\mathrm{H} 1$ antibody. Depletion of RNase $\mathrm{H} 1$ by siRNAs significantly promoted virus replication in mESCs, while overexpression of RNase $\mathrm{H} 1$ suppressed virus replication. Furthermore, expression of the enzyme-dead RNase $\mathrm{H} 1$ mutant failed to inhibit virus replication, further demonstrating that the RNase $\mathrm{H}$ activity is required for this antiviral pathway.

Taken together, the authors revealed a new antiviral pathway in mESCs that is composed of the endogenous RTase and RNase $H$, and gave it a smart name as ERASE, the acronym for endogenous RTase/RNase H-mediated antiviral system, implying that the invading RNA viruses can be erased via the ERASE mechanism. As the vDNA generated by RTase is the key component to recruit RNase $\mathrm{H} 1$ to digest viral RNA, the ERASE mechanism can be regarded as a novel type of nucleic acid-based antiviral defense, which is an addition to the previously known CRISPR/Cas in bacteria and archaea and RNAi antiviral mechanisms in plants and insects. Mechanistically, the ERASE is DNA-mediated RNA cleavage, parallel to the RNA-guided DNA cleavage of CRISPR/Cas system and the RNA-guided RNA cleavage of RNAi pathway.

The existence of DNA form of non-retroviral RNA viruses in mammalian cells has been reported for decades, ${ }^{7,8}$ but its biological significance is not known until now. This study represents the first report to demonstrate the direct antiviral function of these vDNA copies. Very recently, it was reported that the RNA of SARS-CoV-2 can be reverse transcribed into DNA and then integrated into the genome of cultured cells, ${ }^{9}$ though yet controversial. However, the study on SARS-CoV-2 used ectopic overexpression of exogenous RTase and according to the study of Wu et al., it would be highly unlikely that the SARS-CoV-2 RNA

${ }^{1}$ CAS Key Laboratory of Pathogen Microbiology and Immunology, Institute of Microbiology, Chinese Academy of Sciences, Beijing, China. ${ }^{2}$ Chinese Center for Disease Control and Prevention, Beijing, China. ${ }^{凶}$ email: gaof@im.ac.cn 
could be reverse transcribed into DNA by the endogenous RTase in differentiated cells.

Of note, the authors mentioned that they could not show the ERASE activity in long-term cultured human ESCs (hESCs). Such discrepancy may be explained by the fact that long-term cultured hESCs is analogous to the mouse epiblast cells (mEpiSCs) at the post-implantation stage but not $\mathrm{mESCs}$ at the pre-implantation stage. ${ }^{10}$ It will be interesting to evaluate whether this antiviral mechanism works in the naive hESCs, which contain more active ERVs, ${ }^{11}$ and other mammalian ESCs in the future.

Overall, the current work by $\mathrm{Wu}$ et al. demonstrates a novel nucleic acid-based antiviral pathway composed of the endogenous RTase and RNase $\mathrm{H}$. It also unravels the mechanism that the mESCs used to defend against virus infection without the activation of interferon pathway and provides intriguing insights into the relationship among host cells, ERVs and exogenous infective viruses. In view of the wide distribution of RTase and RNase $H$, it will be fascinating to see whether the ERASE mechanism functions in different cell types or organisms. In addition, siRNAs (RNAi) and sgRNAs (CRISPR/Cas) have been widely used in gene knockdown and gene editing in basic and clinical research, and the study by $\mathrm{Wu}$ et al. unraveled the theoretical basis for using artificially designed antisense oligo (ASO) DNA in specific gene knockdown or viral therapy by targeting viral RNAs. It is very commendable that Professor Deyin Guo skillfully named this mechanism as ERASE, which means that the invading virus is "erased".

\section{REFERENCES}

1. tenOever, B. R. Cell Host Microbe 19, 142-149 (2016).

2. Yan, N. \& Chen, Z. J. Nat. Immunol. 13, 214-222 (2012).

3. Horvath, P. \& Barrangou, R. Science 327, 167-170 (2010).

4. Guo, Z., Li, Y. \& Ding, S. W. Nat. Rev. Immunol. 19, 31-44 (2019).

5. Wu, J. et al. Cell Res. 31, 998-1010 (2021).

6. Johnson, W. E. Nat. Rev. Microbiol. 17, 355-370 (2019).

7. Klenerman, P., Hengartner, H. \& Zinkernagel, R. M. Nature 390, 298-301 (1997).

8. Geuking, M. B. et al. Science 323, 393-396 (2009).

9. Zhang, L. et al. Proc. Natl. Acad. Sci. USA 118, e2105968118 (2021).

10. Tesar, P. J. et al. Nature 448, 196-199 (2007).

11. Theunissen, T. W. et al. Cell Stem Cell 19, 502-515 (2016). 